\title{
Interferência da dor neuropática no nível de independência funcional em indivíduos com lesão medular
}

\author{
Interference of neuropathic pain at functional independence \\ level of individuals with spinal cord injury
}

\author{
Interferencia del dolor neuropático em el nível de \\ independencia funcional em indivíduos com lesión espinal
}

\author{
Mayara Cordeiro de Faria ${ }^{1}$, Andréa Souza Rocha ${ }^{2}$, Marinna Coelho \\ Oliveira ${ }^{1}$, Tailliny Damasceno Souza ${ }^{3}$, Francine Aguilera Rodrigues da \\ Silva ${ }^{4}$, Paulo Fernando Lôbo Corrêa ${ }^{4}$
}

\begin{abstract}
1.Fisioterapeuta, Especialista em Saúde Funcional e Reabilitação, Centro Estadual de Reabilitação e Readaptação Dr. Henrique Santillo (CRER), Goiânia-GO, Brasil.

2.Fisioterapeuta, Mestre em Saúde Coletiva, Universidade Federal de Goiás (UFG), Goiânia-Go, Brasil.

3.Discente em Fisioterapia, Pontifícia Universidade Católica de Goiás (PUC-GO), Goiânia-GO, Brasil.

4.Fisioterapeuta, Mestre em Ciências da Saúde, UFG, Goiânia-GO, Brasil.
\end{abstract}

\begin{abstract}
Resumo
Objetivo. Avaliar a interferência da dor neuropática na independência funcional de indivíduos com Lesão Medular Espinal (LME). Método. Estudo quantitativo transversal analítico, realizado em Goiânia-Goiás. Foi aplicado um questionário sociodemográfico e clínico elaborado pelos próprios autores, o Douleur Neuropathique 4 questions (DN4), o Inventário Breve de Dor (IBD) e a Spinal Cord Independence Measure III (SCIM III). Resultados. A amostra foi composta por 57 indivíduos diagnosticados com LME, com predomínio do sexo masculino e média de idade de 37,6 anos. A etiologia predominante foi a traumática, sendo $35 \%$ por acidente de trânsito e a média do tempo de lesão foi 58 meses. Houve prevalência de indivíduos com lesão à nível torácico e com classificação segundo a ASIA Impairment Scale (AIS) A e C. A dor neuropática esteve presente em $74 \%$ dos indivíduos avaliados, com sintomas de formigamento $(88 \%)$ e queimação ( $81 \%$ ) sendo os mais frequentes, somados ao sinal de hipoestesia à picada de agulha $(90 \%)$ e toque $(83 \%)$. A interferência da dor foi maior sobre o humor, enquanto a área menos afetada foi sobre o relacionamento com outras pessoas. Em relação a funcionalidade, indivíduos com dor neuropática apresentaram pontuação inferior do nível funcional em relação aos indivíduos sem dor, entretanto não foi observada correlação significante entre a dor e o nível de independência funcional. Conclusão. Houve pior pontuação relacionada à independência funcional em indivíduos com dor neuropática, principalmente nos itens de autocuidado e respiração/controle de esfíncteres, entretanto não houve correlação significante da dor neuropática e a funcionalidade.
\end{abstract}

Unitermos. Traumatismos da Medula Espinal; Avaliação da Deficiência; Medição da Dor; Reabilitação.

\footnotetext{
Abstract

Objective. To evaluate the interference of neuropathic pain in the functional independence of individuals with Spinal Cord Injury (SCI). Method. Cross-sectional analytical study, carried out in Goiânia-Goiás. A sociodemographic and clinical questionnaire prepared by the authors, the Douleur Neuropathique 4 questions (DN4), the Brief Pain Inventory (IBD) and the Spinal Cord Independence Measure III (SCIM III), were applied. Results. The sample consisted of 57 individuals diagnosed with SCI, with a predominance of males and a mean age of 37.6 years. The predominant etiology was traumatic, 35\% due to traffic accident and the average time of injury was 58 months. There was a prevalence of individuals with injury at the thoracic level and classified according to the ASIA Impairment Scale (AIS) A and C. Neuropathic pain was present in $74 \%$ of the individuals evaluated, with symptoms of tingling ( $88 \%)$ and burning
} 
( $81 \%$ ) being the most frequent, added to the sign of hypoesthesia, needle prick (90\%) and touch $(83 \%)$. The interference of pain was greater on mood, while the least affected area was on relationships with other people. Regarding functionality, individuals with neuropathic pain had lower functional level scores compared to individuals without pain, however no significant correlation was observed between pain and the level of functional independence. Conclusion. There was a worse score related to functional independence in individuals with neuropathic pain, especially in the items of self-care and breathing/sphincter control, however there was no significant correlation between neuropathic pain and functionality.

Keywords. Spinal Cord Injuries; Disability Evaluation; Pain Measurement; Rehabilitation

\section{Resumen}

Objetivo. Evaluar la interferencia del dolor neuropático en la independencia funcional de los individuos con Lesión de la Médula Espinal (LME). Método. Estudio analítico transversal, realizado en Goiânia-Goiás. Se aplicó un cuestionario sociodemográfico y clínico elaborado por los autores, el Douleur Neuropathique 4 questions (DN4), el Inventario Breve de Dolor (EII) y la Spinal Cord Independence Measure (SCIM III). Resultados. La muestra estuvo conformada por 57 individuos diagnosticados de LME, con predominio del sexo masculino y una edad media de 37,6 años. La etiología predominante fue traumática, 35\% por accidente de tráfico y el tiempo medio de lesión fue de 58 meses. Hubo una prevalencia de individuos con lesión a nivel torácico y clasificados según la Escala de Deterioro ASIA (AIS) A y C. El dolor neuropático estuvo presente en el $74 \%$ de los individuos evaluados, siendo los síntomas de hormigueo $(88 \%)$ y ardor $(81 \%)$ los más frecuentes, sumados al signo de hipoestesia, pinchazo de aguja $(90 \%)$ y tacto $(83 \%)$. La interferencia del dolor fue mayor en el estado de ánimo, mientras que el área menos afectada fue en las relaciones con otras personas. En cuanto a la funcionalidad, los individuos con dolor neuropático tuvieron puntuaciones de nivel funcional más bajas en comparación con los individuos sin dolor, sin embargo, no se observó una correlación significativa entre el dolor y el nivel de independencia funcional. Conclusión. Hubo una peor puntuación relacionada con la independencia funcional en los individuos con dolor neuropático, especialmente en los ítems de autocuidado y control de respiración/esfínter, sin embargo no hubo correlación significativa entre el dolor neuropático y la funcionalidad.

Palabras clave. Traumatismos de la Médula Espinal; Evaluación de la Discapacidad; Dimensión del Dolor; Rehabilitación

Trabalho realizado no Centro Estadual de Reabilitação e Readaptação Dr. Henrique Santillo (CRER), Goiânia-GO, Brasil.

Endereço para correspondência: Mayara Cordeiro de Faria. Rua Nepomuceno, Setor Negrão de Lima, Goiânia-GO, Brasil. E-mail: maycfaria.fisio@gmail.com

\section{INTRODUÇÃO}

A Lesão Medular Espinal (LME) é uma condição decorrente da interrupção parcial ou completa da comunicação dos tratos nervosos espinhais abaixo do nível da lesão ${ }^{1}$. O perfil epidemiológico da LME no Brasil apresenta maior prevalência em indivíduos do sexo masculino, na terceira década de vida e com as principais etiologias relacionadas a traumas por projéteis de arma de fogo (PAF) 
e acidentes automobilísticos, sendo a paraplegia completa a condição mais comum².

As alterações provocadas pela LME podem provocar incapacidade funcional e consequentemente comprometer a independência do indivíduo. Em decorrência disso, a saúde pública também é afetada, pois pode aumentar em quase oito vezes mais os custos de saúde em relação ao indivíduo sem LME $^{3}$. Somado a isso, indivíduos com LME apresentam maior dificuldade na obtenção de trabalho remunerado, o que consequentemente gera carência financeira ${ }^{4}$.

Um fator que pode acentuar estes impactos é a presença da dor neuropática, que afeta tanto a vida pessoal quanto a vida social do indivíduo com $\mathrm{LME}^{5}$. Um estudo mostrou que $70 \%$ dos indivíduos com LME adquirem dor neuropática e que destes, existe alto índice de persistência da dor, mesmo após o primeiro ano de lesão6. De forma geral, a dor pode alterar o humor, a dificuldade em lidar com os problemas advindos da lesão e ainda, colaborar para o aparecimento de incapacidades ${ }^{7}$.

Considerando o quanto a dor pode afetar negativamente a independência funcional de indivíduos com LME, o objetivo deste estudo foi avaliar a interferência da dor neuropática na independência funcional de pessoas com LME dos tipos traumáticas e não traumáticas. 


\section{MÉTODO}

\section{Amostra}

Trata-se de um estudo quantitativo transversal analítico, realizado em um centro de reabilitação e readaptação, localizado em Goiânia-GO. Foi aprovado pelo comitê de ética e pesquisa do Centro de Excelência em Ensino, Pesquisa e Projetos Leide das Neves Ferreira (CAAE: 29840420.7.0000.5082).

A amostra foi composta por indivíduos com diagnóstico de LME, que estavam em atendimento ambulatorial ou internados no período de setembro a dezembro de 2020. Foram considerados os seguintes critérios de inclusão: idade mínima de 18 anos; lesões medulares traumáticas ou não traumáticas, completas ou incompletas; qualquer nível e tempo de lesão medular; pacientes em tratamento no centro de reabilitação e readaptação. Os critérios de exclusão foram: indivíduos incapazes de responder instruções verbais; presença de outras doenças neurológicas centrais e/ou periféricas.

\section{Instrumentos}

Um questionário sociodemográfico e clínico, elaborado pelos próprios autores, com dados referentes a: idade, sexo, procedência, índice de massa corporal (IMC), escolaridade, data da lesão, presença de artrodese, tempo de lesão, presença de dor e tempo de início após a lesão, nível, tipo, causa da lesão e sua classificação, segundo a escala fundamentada pela American Spinal Injury Association 
(ASIA), chamada de ASIA Impairment Scale (AIS). A AIS deve ser determinada em $A, B, C, D$ e $E$, onde a primeira se destina a lesões completas e as seguintes para lesões incompletas ${ }^{8}$.

O Douleur Neuropathique 4 questions (DN4) foi utilizado para o rastreio da dor neuropática. Trata-se de um instrumento validado e traduzido para o português no intuito de distinção entre dores neuropáticas e nociceptivas. Na sua validação para o português, atingiu sensibilidade de $100 \%$ e especificidade de 93,2\%, além de propiciar o diagnóstico correto em $96 \%$ dos indivíduos avaliados ${ }^{9}$. Apesar de ser utilizado em diversas condições clínicas, o DN4 mostrou-se confiável na triagem da dor neuropática em indivíduos com LME. O instrumento é composto por 10 itens, dos quais 7 são avaliados por meio dos sintomas e 3 pelo exame físico. Cada item corresponde a 1 ponto, logo a pontuação total varia de 0 a 10, onde 1-3 refere-se a dor nociceptiva e de 4-10 a dor neuropática ${ }^{10}$.

O Inventário Breve de Dor (IBD) foi aplicado para avaliar a intensidade da dor no momento da avaliação, a dor mais e menos intensa e sua média nas últimas 24 horas, assim como, o quanto a dor interfere na atividade geral, humor, habilidade de caminhar, trabalho, relacionamento com outras pessoas, sono e habilidade para apreciar a vida. Esses itens possuem uma pontuação gradual numérica, em uma escala de 0 (nenhuma dor/não interfere) até 10 (pior dor/interferência total) ${ }^{11}$. Outro dado avaliado pelo IBD é a melhora da dor proporcionada por tratamentos 
conservadores ou medicamentosos, variando entre uma escala de 0 a $100 \%$. O IBD foi validado para o português através de um estudo em indivíduos com dor oncológica ${ }^{12}$, porém, pode ser utilizado em indivíduos não oncológicos.

O nível de independência funcional foi avaliado através da Spinal Cord Independence Measure III (SCIM III). Tratase de um instrumento traduzido e validado para a língua portuguesa do Brasil, que apresentou sensibilidade para avaliar as variações funcionais dos indivíduos, reforçando seu uso clínico no gerenciamento do processo de reabilitação de indivíduos com $\mathrm{LME}^{13}$. Foi criado especificamente para avaliar indivíduos com LME e é composto por 17 itens, divididos em três sub-escalas (autocuidado; respiração e controle de esfíncteres; mobilidade). A pontuação total varia de 0 a 100, em que pontuações mais elevadas refletem níveis mais altos de independência ${ }^{13,14}$.

\section{Procedimentos}

O recrutamento e triagem dos possíveis participantes foram realizados por meio de uma busca ativa nas agendas dos profissionais que atendiam os ambulatórios de reabilitação da instituição. Para os possíveis participantes em regime de internação, diariamente os pesquisadores acessavam a lista de pacientes internados na instituição para identificá-los. Após este levantamento, os critérios de inclusão e exclusão poderiam ser verificados por meio da análise dos prontuários eletrônicos. Finalmente, os indivíduos foram abordados antes e/ou após os seus 
atendimentos (quando em regime ambulatorial) ou no leito (quando em regime de internação). Nesta abordagem eles foram esclarecidos sobre a pesquisa e convidados a participar.

Os indivíduos que aceitaram participar, assinaram 0 Termo de Consentimento Livre e Esclarecido (TCLE) e a coleta de dados foi realizada em seguida, com os instrumentos preenchidos pelos próprios pesquisadores. Após o término da coleta, os pesquisadores analisaram o prontuário do indivíduo, a fim de verificar algumas informações clínicas, como o nível e tipo de lesão, assim como a classificação segundo a AIS. Todos os procedimentos relacionados a análise dos prontuários foram realizados após a autorização da instituição e mediante o Termo de Compromisso de Utilização de Dados.

\section{Análise estatística}

A análise estatística inicialmente contou com a análise descritiva dos dados, sendo média, desvio padrão e intervalo de confiança para as variáveis escalares e em frequência e porcentagem para as variáveis categóricas. Para as comparações por grupos utilizou-se dos testes U Mann Whitney e Kruskal-Wallis, seguindo análise prévia de parametrização pelo teste de Shapiro-Wilk. A análise de associação foi realizada pelo teste qui-quadrado e em proporções $2 \times 2$ calculou-se a razão de chances (odds ratio). Estatística realizada no Statistical Package for the Social 
Sciences versão 26.0 adotando significância com valor de $\mathrm{p} \leq 0,05$.

\section{RESULTADOS}

Foram avaliados 67 indivíduos com LME, porém 10 foram excluídos, sete não possuíam dados nos prontuários relacionados ao nível de lesão e/ou classificação segundo a AIS; dois sofreram Traumatismo Cranioencefálico (TCE) concomitante a LME e um teve Acidente Vascular Encefálico (AVC) antes da LME, portanto, a amostra final foi composta por 57 indivíduos com LME.

Em relação as características sociodemográficas da amostra (Tabela 1), a idade variou de 18 a 70 anos (média $37,6 \pm 13,5)$, a maioria era do sexo masculino, residente em Goiânia ou região metropolitana. Em relação à escolaridade, não houve participantes analfabetos, a maioria tinha nível médio completo ou nível fundamental incompleto.

Considerando as características clínicas, a etiologia predominante foi a traumática, principalmente por acidente de trânsito e projéteis de arma de fogo (PAF). Os outros tipos de traumas relatados foram: quedas $(7 \%)$, acidente em escorregador aquático (5\%); queda de objeto sobre o indivíduo (4\%); mergulho em águas rasas (MAR) (4\%) ou trauma cirúrgico (2\%). Dentre as lesões compressivas, 50\% foram por hérnia discal e $50 \%$ por tumores. 
Tabela 1. Características sociodemográficas e clínicas da amostra.

\begin{tabular}{|c|c|c|}
\hline Variável & $\begin{array}{l}\text { Frequência Absoluta } \\
\text { (n) }\end{array}$ & $\begin{array}{c}\text { Frequência } \\
\text { Relativa (\%) }\end{array}$ \\
\hline \multicolumn{3}{|l|}{ Sexo } \\
\hline Feminino & 14 & 25 \\
\hline Masculino & 43 & 75 \\
\hline \multicolumn{3}{|l|}{ Local de residência } \\
\hline Capital (Goiânia) & 38 & 67 \\
\hline Região Metropolitana & 12 & 21 \\
\hline Interior & 7 & 12 \\
\hline \multicolumn{3}{|l|}{ Escolaridade } \\
\hline Analfabeto & 0 & 0 \\
\hline Fundamental incompleto & 16 & 28 \\
\hline Fundamental completo & 3 & 5 \\
\hline Médio incompleto & 7 & 12 \\
\hline Médio completo & 19 & 33 \\
\hline Superior incompleto & 3 & 5 \\
\hline Superior completo & 7 & 12 \\
\hline Mestrado & 2 & 4 \\
\hline \multicolumn{3}{|l|}{ IMC } \\
\hline Magreza $(<18,5)$ & 2 & 4 \\
\hline Normal $(18,6$ a 24,9$)$ & 23 & 40 \\
\hline Sobrepeso (25 a 29,9$)$ & 11 & 19 \\
\hline Obesidade $(30 \mathrm{a}>40)$ & 9 & 16 \\
\hline Não informado & 12 & 21 \\
\hline \multicolumn{3}{|l|}{ Tipo de lesão } \\
\hline Traumática & 46 & 81 \\
\hline Não traumática & 11 & 19 \\
\hline \multicolumn{3}{|l|}{ Etiologia } \\
\hline PAF & 14 & 25 \\
\hline Acidente de trânsito & 20 & 35 \\
\hline MAR & 2 & 4 \\
\hline Infecciosas & 3 & 5 \\
\hline Compressão & 8 & 14 \\
\hline Outras & 10 & 18 \\
\hline \multicolumn{3}{|l|}{ Nível geral da lesão } \\
\hline Cervical alta (C1-C4) & 7 & 12 \\
\hline Cervical baixa (C5-C7) & 14 & 25 \\
\hline Torácica alta (T1-T6) & 14 & 25 \\
\hline Torácica baixa (T7-T12) & 12 & 21 \\
\hline Lombar (L1-L5) & 10 & 18 \\
\hline \multicolumn{3}{|l|}{ Artrodese na coluna espinal } \\
\hline Sim & 34 & 60 \\
\hline Não & 23 & 40 \\
\hline \multicolumn{3}{|l|}{ Classificação da AIS } \\
\hline A & 19 & 33 \\
\hline $\mathrm{B}$ & 5 & 9 \\
\hline $\mathrm{C}$ & 18 & 32 \\
\hline $\mathrm{D}$ & 13 & 23 \\
\hline $\mathrm{E}$ & 2 & 4 \\
\hline \multicolumn{3}{|l|}{ Tempo de lesão } \\
\hline 0 a 3 meses & 1 & 2 \\
\hline 3 a 6 meses & 1 & 2 \\
\hline 6 a 12 meses & 6 & 11 \\
\hline Acima de 12 meses & 49 & 86 \\
\hline \multicolumn{3}{|l|}{ Início da dor após a lesão } \\
\hline 0 a 3 meses & 41 & 72 \\
\hline 3 a 6 meses & 5 & 9 \\
\hline 6 a 12 meses & 0 & 0 \\
\hline Acima de 12 meses & 1 & 2 \\
\hline Ausência de dor & 10 & 18 \\
\hline
\end{tabular}

IMC: Índice de Massa Corporal; AIS: ASIA Impairment Scale; PAF: Projéteis de arma de fogo; MAR: Mergulho em águas rasas. 
Em relação ao nível de lesão, 37\% foram a nível cervical, $46 \%$ torácica e $18 \%$ lombar. A gravidade da lesão foi graduada por meio da AIS e as mais prevalentes foram do tipo A e C. O tempo de lesão variou de um mês a 31,5 anos (58 $\pm 67,6$ meses).

A grande maioria dos indivíduos (82\%) apresentaram alguma dor secundária a $L M E$, sendo mais comum o início imediato após a lesão.

A grande maioria dos indivíduos avaliados (74\%) apresentaram dor neuropática, de acordo com o DN4. As tabelas 2 e 3 caracterizam apenas a parte da amostra, na qual, a dor neuropática esteve presente (42 indivíduos).

A variabilidade da apresentação da dor neuropática, de acordo com a DN4, variou de 4 a 9 pontos (média de $4,7 \pm 2,7)$. As formas de apresentação presentes em mais de $80 \%$ dos casos foram hipoestesia à picada de agulha, seguido por formigamento, hipoestesia ao toque e queimação. A forma menos frequente foi a coceira (14\%) (Tabela 2).

Tabela 2. Ocorrência e formas de apresentação da dor neuropática segundo o Douleur Neuropathique 4 questions (DN4).

\begin{tabular}{|c|c|c|}
\hline Variável & Frequência Absoluta (n) & $\begin{array}{c}\text { Frequência Relativa } \\
(\%)\end{array}$ \\
\hline \multicolumn{3}{|l|}{ Tipo de dor } \\
\hline Dor neuropática & 42 & 74 \\
\hline Dor nociceptiva & 5 & 9 \\
\hline Ausência de dor & 10 & 17 \\
\hline \multicolumn{3}{|c|}{ Sinais e sintomas na dor neuropática $(n=42)$} \\
\hline Queimação & 34 & 81 \\
\hline Sensação de frio dolorosa & 14 & 33 \\
\hline Choque elétrico & 27 & 64 \\
\hline Formigamento & 37 & 88 \\
\hline Alfinetada e agulhada & 24 & 57 \\
\hline Adormecimento & 30 & 71 \\
\hline Coceira & 6 & 14 \\
\hline Hipoestesia ao toque & 35 & 83 \\
\hline Hipoestesia à picada de agulha & 38 & 90 \\
\hline Dor à escovação & 11 & 26 \\
\hline
\end{tabular}


Dos 42 indivíduos com dor neuropática, 83\% relataram presença de dor no dia da avaliação. A topografia da dor neuropática foi distribuída entre os membros e tronco (50\%), apenas nos membros inferiores (MMII) (31\%), apenas tronco (12\%) e tanto nos MMII quanto nos membros superiores (MMSS) (7\%).

Considerando a interferência da dor nos diversos aspectos da vida cotidiana, a maior interferência foi sobre o humor, enquanto a menor interferência foi sobre 0 relacionamento com outras pessoas (Tabela 3). Apesar do IBD avaliar a interferência da dor na habilidade de caminhar e no trabalho, foi necessário excluir esses itens da avaliação, pois grande parte dos indivíduos avaliados não conseguiam caminhar e a única forma de locomoção deles era em cadeira de rodas.

Tabela 3. Interferência da dor neuropática segundo o Inventário Breve de Dor (IBD). $\mathrm{N}=42$.

\begin{tabular}{lcc}
\hline Variável & Média & Desvio Padrão (DP) \\
\hline Intensidade da dor (0-10) & & $\pm 2,9$ \\
Pior dor nas últimas 24 horas & 6,6 & $\pm 2,7$ \\
Dor mais fraca nas últimas 24 horas & 3,3 & $\pm 2,7$ \\
Média da dor nas últimas 24 horas & 5,5 & $\pm 3,1$ \\
$\quad$ Dor no momento da avaliação & 4,6 & \\
\hline Melhora da dor proporcionada por tratamentos conservadores & & $\pm 40,2$ \\
ou medicamentosos (0-100\%) & 46,9 & $\pm 3,5$ \\
\hline Interferência da dor (0-10) & 4,4 & $\pm 3,6$ \\
Atividade Geral & 5,6 & $\pm 3,9$ \\
Humor & 3,5 & $\pm 3,9$ \\
Relacionamentos & 4,2 & $\pm 3,9$ \\
Sono & 4,5 & \\
Habilidade de apreciar a vida & & \\
\hline
\end{tabular}

Ao avaliarmos a pontuação total da SCIM III, observouse que os indivíduos com dor neuropática possuíram pior pontuação em relação aos indivíduos do grupo sem dor 
neuropática, formado pelos indivíduos com dor nociceptiva e com ausência de dor $(n=15)$. A funcionalidade de indivíduos com dor neuropática foi pior nas subescalas: autocuidado; respiração e controle de esfíncteres, comparado aos indivíduos do grupo sem dor neuropática. Já o score da subescala de mobilidade, foi pior nos indivíduos sem dor neuropática (Tabela 4).

Em relação à classificação pela AIS e o nível de lesão, os indivíduos com dor neuropática, em sua maioria, possuíam classificação AIS C e D e lesão à nível torácico. Já os indivíduos do grupo sem dor neuropática, possuíam predomínio de AIS A e B e o nível de lesão também foi a torácica (Tabela 4).

Tabela 4. Funcionalidade dos indivíduos com LME segundo a Spinal Cord Independence Measure III (SCIM III). Média (DP).

\begin{tabular}{|c|c|c|c|c|}
\hline & $\begin{array}{c}\text { Total } \\
(0-100)\end{array}$ & $\begin{array}{c}\text { Autocuidado } \\
(0-20)\end{array}$ & $\begin{array}{c}\text { Respiração e controle } \\
\text { dos esfíncteres } \\
(0-40)\end{array}$ & $\begin{array}{c}\text { Mobilidade } \\
\quad(0-40)\end{array}$ \\
\hline $\begin{array}{l}\text { Dor neuropática }(n=42) \\
\text { AIS A e } B=33 \% \\
\text { AIS C e } D=64 \% \\
\text { AIS } E=2 \% \\
\text { Cervical }=40 \% \\
\text { Torácica }=45 \% \\
\text { Lombar }=17 \%\end{array}$ & $57 \pm 21,3$ & $14,8 \pm 6,4$ & $24,3 \pm 8$ & $17,9 \pm 10,1$ \\
\hline $\begin{array}{l}\text { Dor nociceptiva }(n=5) \\
\text { AIS A e } B=40 \% \\
\text { AIS C e } D=40 \% \\
\text { AIS E }=20 \% \\
\text { Cervical }=0 \% \\
\text { Torácica }=40 \% \\
\text { Lombar }=60 \%\end{array}$ & $73,8 \pm 15$ & $17 \pm 2$ & $31,2 \pm 6,1$ & $25,6 \pm 10,4$ \\
\hline $\begin{array}{l}\text { Ausência de qualquer tipo de dor } \\
(n=10) \\
\text { AIS A e B }=80 \% \\
\text { AIS C e } D=20 \% \\
\text { AIS E }=0 \% \\
\text { Cervical }=50 \% \\
\text { Torácica }=50 \% \\
\text { Lombar }=0 \%\end{array}$ & $50,6 \pm 18,7$ & $14,2 \pm 6,1$ & $23,2 \pm 7,9$ & $13,2 \pm 5,8$ \\
\hline $\begin{array}{l}\begin{array}{l}\text { Ausência de dor neuropática } \\
(n=15)\end{array} \\
\text { AIS A e } B=67 \% \\
\text { AIS C e } D=27 \% \\
\text { AIS } E=7 \% \\
\text { Cervical }=33 \% \\
\text { Torácica }=47 \% \\
\text { Lombar }=20 \%\end{array}$ & $58,3 \pm 20,4$ & $15,1 \pm 5,2$ & $25,9 \pm 8,1$ & $17,3 \pm 9,4$ \\
\hline
\end{tabular}


Apesar da pontuação total da funcionalidade avaliada pela SCIM III nos indivíduos com LME ser inferior naqueles com dor neuropática (média $=56,98$ ) em relação àqueles que não possuem dor neuropática (média=58,33), não foi observada diferença significante (Tabela 5).

Tabela 5. Comparação do nível funcional de indivíduos com dor neuropática e ausência da mesma. $\mathrm{N}=57$.

\begin{tabular}{llcccc}
\hline & Classificação da dor & N & Média & $\begin{array}{c}\text { Desvio } \\
\text { Padrão }\end{array}$ & p valor \\
\hline \multirow{2}{*}{ SCIM III (total) } & Dor neuropática & 42 & 56,98 & 21,33 & \\
& $\begin{array}{l}\text { Ausência de dor } \\
\text { neuropática }\end{array}$ & 15 & 58,33 & 20,43 & 0,831 \\
& Dor neuropática & 42 & 14,81 & 6,39 & \\
Autocuidado & Ausência de dor & 15 & 15,13 & 5,18 & 0,861 \\
& neuropática & & & & \\
\hline $\begin{array}{l}\text { Respiração e controle } \\
\text { de esfíncteres }\end{array}$ & Dor neuropática & 42 & 24,29 & 7,96 & 0,521 \\
& $\begin{array}{l}\text { Ausência de dor } \\
\text { neuropática }\end{array}$ & 15 & 25,87 & 8,11 & \\
\hline \multirow{2}{*}{ Mobilidade } & Dor neuropática & 42 & 17,88 & 10,05 & 0,855 \\
& Ausência de dor & 15 & 17,33 & 9,45 & \\
\hline & neuropática & & & & \\
\hline
\end{tabular}

\section{DISCUSSÃO}

Os resultados deste estudo demonstram que a maioria dos indivíduos com LME são do sexo masculino e adultos jovens. Dados similares aos encontrados na literatura, na qual a prevalência da LME no sexo masculino variou de $68 \%{ }^{15}$ a $91 \%{ }^{16}$ e a média de idade variou entre $32,2^{17}$ e 37,9 anos $^{16}$, demonstrando maior prevalência na quarta década de vida. Tais dados podem ser atribuídos ao fato de 
indivíduos mais jovens serem mais ativos, propiciando maior tendência a lesões.

Neste estudo houve prevalência de indivíduos com lesões traumáticas, e em sua maioria foram relacionadas a acidentes de trânsito. Esse dado confirma dados da literatura, os quais apresentam que as causas traumáticas são mais comuns ${ }^{18}$, e considerando essa etiologia, os acidentes de trânsito ${ }^{19}$ e ferimentos por PAF $^{20}$ são os mais frequentes, podendo ser relacionados à maior exposição à violência urbana ${ }^{19}$.

A maioria dos indivíduos avaliados neste estudo possuíam lesões à nível torácico, corroborando com dados da literatura, que demonstraram que na LME o nível mais acometido é o torácico ${ }^{15,21}$. Esses dados podem ser associados ao fato da coluna torácica ser a região mais extensa da coluna vertebral, o que a deixa mais suscetível a lesões ${ }^{22}$.

No presente estudo, houve prevalência de indivíduos classificados em AIS A e com lesões por acidentes de trânsito, o que concorda com a literatura. Estudos mostraram que a maioria dos indivíduos possuem lesão classificada em AIS $A^{23,24}$ e que em acidentes automobilísticos as lesões classificadas em AIS A são as mais frequentes ${ }^{25}$. Sabe-se que os motociclistas estão predispostos a maior gravidade clínica quando feridos por acidentes de trânsito, o que pode relacionar à prevalência apresentada anteriormente. 
Neste estudo, houve prevalência de $74 \%$ de dor neuropática nos indivíduos com LME e em sua maioria o início foi precoce (até três meses após a LME). Os mecanismos subjacentes à dor da LME permanecem mal compreendidos ${ }^{1}$. A prevalência de dor em indivíduos com LME varia de $64 \%$ a $82 \%$, com o início em dias ou até anos após a lesão, com maior frequência nos seis primeiros meses, destacando também que a dor neuropática no nível da lesão tende aparecer mais precocemente, enquanto a dor abaixo do nível da lesão inicia-se tardiamente ${ }^{7}$.

Este estudo mostrou que as formas de apresentação da dor neuropática presentes em mais de $80 \%$ dos indivíduos, com a utilização do DN4, foram: queimação e formigamento, associados à hipoestesia ao toque e hipoestesia à picada de agulha. As formas de apresentação da dor neuropática mais frequentes encontradas foram: queimação e formigamento, associados à hipoestesia ao toque ${ }^{10}$, corroborando com este estudo. Uma das características da LME é a perda de sensibilidade, o que pode estar associado ao fato de boa parte da amostra ter apresentado hipoestesia ao toque e à picada de agulha.

A média da intensidade da dor neste estudo, correspondeu à 5,5 pontos. Em uma escala de zero a dez, a média da intensidade da dor apresentada foi maior que cinco pontos, o que corrobora com os resultados encontrados neste estudo ${ }^{5}$.

O humor foi o principal aspecto da vida cotidiana afetado pela dor, entretanto, deve-se ponderar não só as 
questões relacionadas à dor, mas sim todas as complicações relacionadas à LME que podem influenciar tanto na vida cotidiana quanto na baixa funcionalidade. Além da presença de dor, o comprometimento da pele, a presença de espasticidade, um IMC alto e a mobilidade reduzida podem levar a um pior nível de independência funcional ${ }^{26,27 .}$

Em relação a um possível impacto desta dor neuropática na funcionalidade, não foi encontrado neste estudo diferença significante entre o grupo que apresentava e o que não apresentava dor neuropática. Isso pode ser atribuído ao fato da funcionalidade em indivíduos com LME sofrer grande influência de outros aspectos não relacionados à presença da dor neuropática, como a classificação da lesão segundo a escala AIS, bem como o nível e tipo de lesão. Neste estudo, a maioria dos indivíduos com dor neuropática foram classificados em AIS C e D, enquanto a maioria dos indivíduos sem dor neuropática foram classificados em AIS A e $B$, dessa forma, apesar do último grupos não possuir dor neuropática, eles são menos funcionais pela própria classificação da lesão.

Os indivíduos com LME apresentaram pontuação média de 55 pontos na SCIM III, independente da presença de dor. Pontuação aproximada ao deste estudo (57 pontos), o que reforça a possibilidade que indivíduos com dor neuropática possuem um nível de independência funcional similar aos que não a possuem ${ }^{28}$.

Na tentativa de analisar a influência da dor neuropática na funcionalidade de indivíduos com LME as principais 
limitações foram a presença de fatores confundidores como: o nível e tipo de lesão, assim como sua classificação pela escala AIS. Outra limitação a se destacar foi a diferença na quantidade de indivíduos com dor neuropática em relação àqueles que não a possuem, o que impossibilitou a análise comparativa dos dois grupos. Limitação que não foi possível de ser evitada devido à alta prevalência de dor neuropática nesta população. Dessa forma, sugere-se novos estudos com grupos mais homogêneos.

\section{CONCLUSÃO}

Observa-se que grande parte dos indivíduos com LME possuem dor neuropática e seu início ocorre principalmente nos primeiros três meses após a lesão. Indivíduos com dor neuropática possuíram pior pontuação relacionada à independência funcional comparado aos indivíduos sem dor neuropática, principalmente relacionada ao autocuidado e respiração/controle de esfíncteres, entretanto não houve correlação significativa da dor e a funcionalidade. Em relação ao aspecto da vida cotidiana que sofre maior interferência da dor foi o humor.

\section{REFERÊNCIAS}

1.Shiao R, Lee-Kubli CA. Neuropathic pain after spinal cord injury: Challenges and research perspectives. Neurotherapeutics 2018;15:635-53. https://doi.org/10.1007/s13311-018-0633-4

2.Barbetta DC, Smanioto TR, Poletto MF, Ferreira RFA, Lopes ACG, Casaro FM, et al. Spinal cord injury epidemiological profile in the Sarah Network of Rehabilitation Hospitals - a Brazilian population sample. Spinal Cord Ser Cases 2018;2-7. https://doi.org/10.1038/s41394-018$\underline{0049-8}$ 
3.Chan BCF, Cadarette SM, Wodchis WP, Krahn MD, Mittmann N. The lifetime cost of spinal cord injury in Ontario, Canada: A populationbased study from the perspective of the public health care payer. J Spinal Cord Med 2019;42:184-93.

http://dx.doi.org/10.1080/10790268.2018.1486622

4.Andrade VS, Faleiros F, Balestrero LM, Romeiro V, Santos CB. Participação social e autonomia pessoal de indivíduos com lesão medular. Rev Bras Enferm 2019;72:250.

http://dx.doi.org/10.1590/0034-7167-2018-0020

5.Aquarone RL, Faro ACM, Nogueira PC. Central neuropathic pain: implications on quality of life of spinal cord injury patients. Rev Dor 2015;16:280-4. http://dx.doi.org/10.5935/1806-0013.20150057

6.Posso IP, Palmeira CCA, Vieira ÉBM. Epidemiology of neuropathic pain. Rev Dor 2016;17:11-4. http://dx.doi.org/10.5935/18060013.20160039

7. Miguel M, Kraychete DC. Dor no Paciente com Lesão Medular: Uma Revisão. Rev Bras Anestesiol 2009;59:350-7. http://dx.doi.org/10.1590/S0034-70942009000300011

8. Rouanet C, Reges D, Rocha E, Gagliardi V, Silva GS. Traumatic spinal cord injury: current concepts and treatment update. Arq Neuropsiquiatr 2017;75:387-93. http://dx.doi.org/10.1590/0004$282 \times 20170048$

9.Santos JG, Brito JO, Andrade DC, Kaziyama VM, Ferreira KA, Souza $I$, et al. Translation to Portuguese and Validation of the Douleur Neuropathique 4 Questionnaire. J Pain 2010;11:484-90.

https://doi.org/10.1016/i.jpain.2009.09.014

10. Hallström H, Norrbrink C. Screening tools for neuropathic pain: Can they be of use in individuals with spinal cord injury? Pain 2011;152:772-9. https://doi.org/10.1016/j.pain.2010.11.019

11. Moura CDC, Chaves ECL, Chianca TCM, Ruginsk SG, Nogueira DA, Iunes DH. Effects of auricular acupuncture on chronic pain in people with back musculoskeletal disorders: a randomized clinical trial. Rev EsC Enferm USP 2019;53:1-9. https://doi.org/10.1590/s1980$\underline{220 \times 2018009003418}$

12.Ferreira KA, Teixeira MJ, Mendonza TR, Cleeland CS. Validation of brief pain inventory to Brazilian patients with pain. Supp Care Cancer 2011;19:505-11. https://doi.org/10.1007/s00520-010-0844-7

13. Riberto M, Tavares DA, Rimoli JRJ, Castineira CP, Dias RV, Franzoi $A C$, et al. Validation of the Brazilian version of the Spinal Cord Independence Measure III. Arq Neuropsiquiatr 2014;72:439-44. https://doi.org/10.1590/0004-282X20140066

14.Ilha J, Avila LCM, Santo CCE, Swarowsky A. Tradução e adaptação transcultural da versão brasileira da Spinal Cord Independece Measure - Self-Reported Version (brSCIM-SR). Rev Bras Neurol 2016;52:2-17. http://files.bvs.br/upload/S/01018469/2016/v52n1/a5457.pdf

15.Soares CFA, Andrade PHM, Muller KTC, Santos SJ. Aspectos sociodemográficos e qualidade de vida de pessoas com Traumatismo da medula espinal. Rev Neurocienc 2020;28:1-14. https://doi.org/10.34024/rnc.2020.v28.10363 
16. Rabeh SAN, Caliri MHL. Capacidade funcional em indivíduos com lesão de medula espinhal. Acta Paul Enferm 2010;23:321-7. https://doi.org/10.1590/S0103-21002010000300002

17. Cardoso FL, Porto IP, Carvalho HP, Ferrari EP. Fatores associados à satisfação sexual de homens com lesão medular. Fisioter Pesqui 2018;25:35-42. https://doi.org/10.1590/1809-2950/16742625012018

18.Coura AS, Enders BC, França ISX, Vieira CENK, Dantas DNA, Menezes DJC. Capacidade de autocuidado e sua associação com os fatores sociodemográficos de pessoas com lesão medular. Rev Esc Enferm USP 2013;47:1154-62. https://doi.org/10.1590/S0080623420130000500020

19.Baldassin V, Lorenzo C, Shimizu HE. Tecnologia assistiva e qualidade de vida na tetraplegia: abordagem bioética. Rev Bioética 2018;26:574-86. https://doi.org/10.1590/1983-80422018264276

20.Vall J, Costa CMC, Santos TJT, Costa SBC. Neuropathic pain characteristics in patients from Curitiba (Brazil) with spinal cord injury. Arq Neuropsiquiatr 2011;69:64-8. https://doi.org/10.1590/S0004$\underline{282 \times 2011000100013}$

21.Cavalcante ES, Júnior JMP, Freire ILS, Cavalcante CAA, Miranda FAN. Representações sociais de pescadores com lesão medular: repercussões e trajetória de vida. Rev Bras Enferm 2017;70:139-45. https://doi.org/10.1590/0034-7167-2016-0436

22.Santos RA, Almeida MLO, Silva MF. Perfil clínico e epidemiológico de pacientes com traumatismo raquimedular. Fisioter Bras 2013;14:215-20. http://dx.doi.org/10.33233/fb.v14i3.394

23.Barrera-Chacon JM, Mendez-Suarez JL, Jáuregui-Abrisqueta ML, Palazon R, Barbara-Bataller E, García-Obrero I. Oxycodone improves pain control and quality of life in anticonvulsantpretreated spinal cordinjured patients with neuropathic pain. Spinal Cord 2011;49:36-42. https://doi.org/10.1038/sc.2010.101

24. Mota D, Ribeiro MB. Qualidade de vida em portadores de lesão medular com úlceras por pressão. Enferm Glob 2016;42:22-30. http://scielo.isciii.es/pdf/eg/v15n42/pt clinica2.pdf

25. Halvorsen A, Pettersen AL, Nilsen SM, Halle KK, Schaanning EE, Rekand T. Epidemiology of traumatic spinal cord injury in Norway in 2012 - 2016: a registry-based cross-sectional study. Spinal Cord 2019;57:331-8. https://doi.org/10.1038/s41393-018-0225-5

26.Ackerman P, Morrison SA, McDowell S, Vazquez L. Using the Spinal Cord Independence Measure III to measure functional recovery in a post-acute spinal cord injury program. Spinal Cord 2010;48:380-7. https://doi.org/10.1038/sc.2009.140

27. Faria MC, Menezes GS, Morais LA. Fatores que interferem na qualidade de vida de indivíduos com lesão medular traumática: uma revisão de literatura. Rev Mov 2020;13:285-96. https://www.revista.ueg.br/index.php/movimenta/article/view/9568/7805 28. Kee KM, Mohamad NZ, Koh PPW, Yeo JPT, Ng YS, Kam JC, et al. Return to work after spinal cord injury: a Singaporean pilot communitybased rehabilitation program. Spinal Cord 2020;1-8. https://doi.org/10.1038/s41393-020-0459-x 